\title{
MeV Helium Ion Imaging of Gold Nanoparticles in Whole Cells
}

\author{
Chen Xiao,* Ren Minqin, ${ }^{*}$ Chen Ce-Belle, ${ }^{*}$ CNB Udalagama,* AA Bettiol,* Fong Kah Ee**, \\ Lanry Yung Lin Yue**, and F Watt* \\ * Centre for Ion Beam Applications, Dept of Physics, National University of Singapore, Singapore \\ 117542 \\ ** Dept of Chemical and Biomedical Engineering, National University of Singapore.
}

Observation of the interior structure of cells and sub-cellular organelles at high spatial resolutions is important for determining the functioning mechanisms of biological cells. Conventional optical microscopies have resolutions limited to around $300 \mathrm{~nm}$ due to the diffraction limits of light, and the new super-resolution techniques such as Stimulated Emission Depletion microscopy (STED) have stringent requirements that limit their application areas. Electron microscopy has an important role to play, but is only useful when imaging very thin sections due to electron/electron large angle scattering within the sample. Our results indicate that microscopy using $\mathrm{MeV}$ ions has high potential for the imaging of thick samples, e.g. whole cells, at high spatial resolutions. The reason for this is that $\mathrm{MeV}$ ions (e.g. protons or alpha particles (helium ions)) maintain a straight trajectory when traversing material, therefore preserving spatial resolution. The interaction of $\mathrm{MeV}$ ions with matter is mainly through ion/electron collisions. Due to the high mass mismatch with electrons, ions suffer very low energy transfer for each collision and as a result thousands of collisions can occur before they stop. In addition, there is very little primary ion scattering, and therefore the $\mathrm{MeV}$ proton and alpha particle paths are characterised by a straight, deep penetration into the material [1]. As well as ion electron scattering, there is also a much smaller probability of the $\mathrm{MeV}$ ion undergoing elastic scattering from an atomic nucleus. In these nuclear collisions the ion can be backscattered (Rutherford backscattering spectrometry - RBS), and by measuring the energy of the backscattered ion, the target atom can be identified. RBS is particularly efficient at identifying and measuring heavy metals in low mass matrices such as organic materials.

The Centre for Ion Beam Applications, National University of Singapore has recently built up a high resolution single cell imaging facility with the ability of focusing for MeV proton and alpha particle beams down to $30 \mathrm{~nm}$ spot sizes [2]. The facility incorporates a variety of techniques, including Scanning Transmission Ion Microscopy (STIM) and Rutherford Backscattering Spectroscopy (RBS), with the microanalytical technique of Particle Induced X-ray Emission (PIXE) being added at a later stage. STIM is a technique which relies on the measurement of the energy loss of transmitted ions. $\mathrm{MeV}$ helium ions or protons are energetic enough to pass through single whole cells, and by measuring the energy loss of the transmitted ions, a structural image can be assembled which has high density contrast.

Gold nanoparticles have great potential uses for medical diagnostics and drug delivery, as tracers and for other biological applications. Although Transmission Electron Microscopy (TEM) has been very successful in identifying nanoparticles in thin cellular sections, it remains difficult to resolve the gold nanoparticles at nanoscale resolution in the whole cell. In this work, we have used STIM and RBS to image normal human lung fibroblast cells (MRC-5) cultured in an environment of $50 \mathrm{~nm}$ gold nanoparticles. Cells were grown on $100 \mathrm{~nm}$ silicon nitride windows in cell culture medium (RPMI supplemented with fetal bovine serum) containing $50 \mathrm{~nm}$ gold nanoparticles $(0.2$ $\mathrm{nM})$. Following exposure to the nanoparticles for 72 hours, the cells were critically point dried. The STIM measurements were carried out using a silicon surface barrier ion detector positioned behind the cell, and the RBS measurements were carried out using an annular surface barrier detector in front of the cell.

The STIM image in Fig. 1 shows the structural view of the whole cell cultured in a gold nanoparticle environment, and Fig. 2 shows a similar control cell grown in normal conditions. The 
resolution in both these cases is around $30 \mathrm{~nm}$, and the helium ion energy was $2 \mathrm{MeV}$. The RBS image (see Fig. 3) displays the distribution of gold in the cells, and since RBS requires a greater current density, is currently limited to a resolution of around 200nm. Fig. 4 shows the off-axis STIM image of the cell at a resolution of $80 \mathrm{~nm}$, which highlights the dense clusters. Gold clusters identified in the RBS image are distributed outside the cell nucleus, and are highly correlated with the STIM images.

Work is currently in progress to improve the spatial resolutions of $\mathrm{MeV}$ helium ion microscopy to the nanometer level by increasing the ion source brightness, and this will enable structural imaging of whole cells at nanometer dimensions, and elemental mapping below $100 \mathrm{~nm}$. In addition, by taking several images at varying angles, 3D tomographic imaging at nanometer resolutions is feasible due to the increased depth of focus of ion beams.

\section{References}

[1] Udalagama et al., Physical Review B, 2009. 80(22): p. 224107.

[2] Watt, F., et al. Nuclear Instruments and Methods in Physics Research Section B: Beam Interactions with Materials and Atoms. In Press, Accepted Manuscript.

[3] This study was supported by the Singapore Bioimaging Consortium (SBIC), ASTAR grant number SBIC RP C-013/2007.
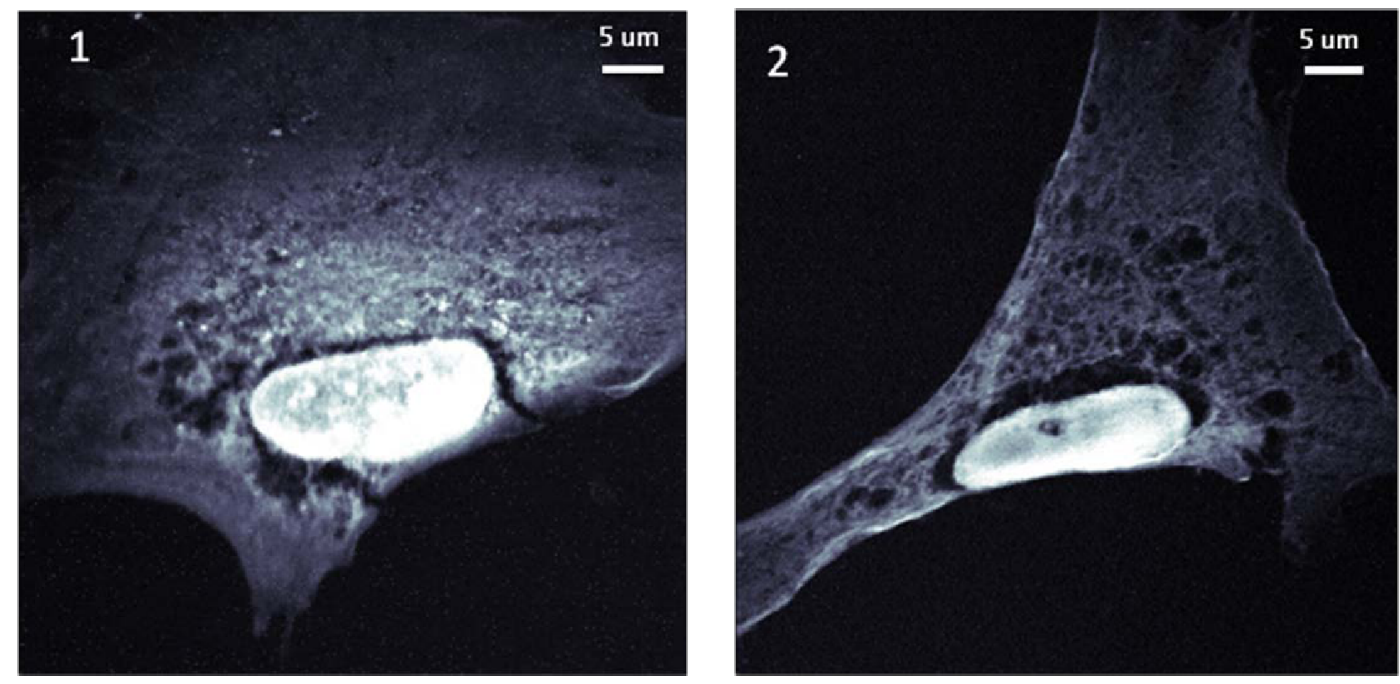

Fig. 1: STIM of cell grown in culture medium containing 50nm Gold nanoparticles.

Fig. 2: STIM of cell grown in standard culture medium.
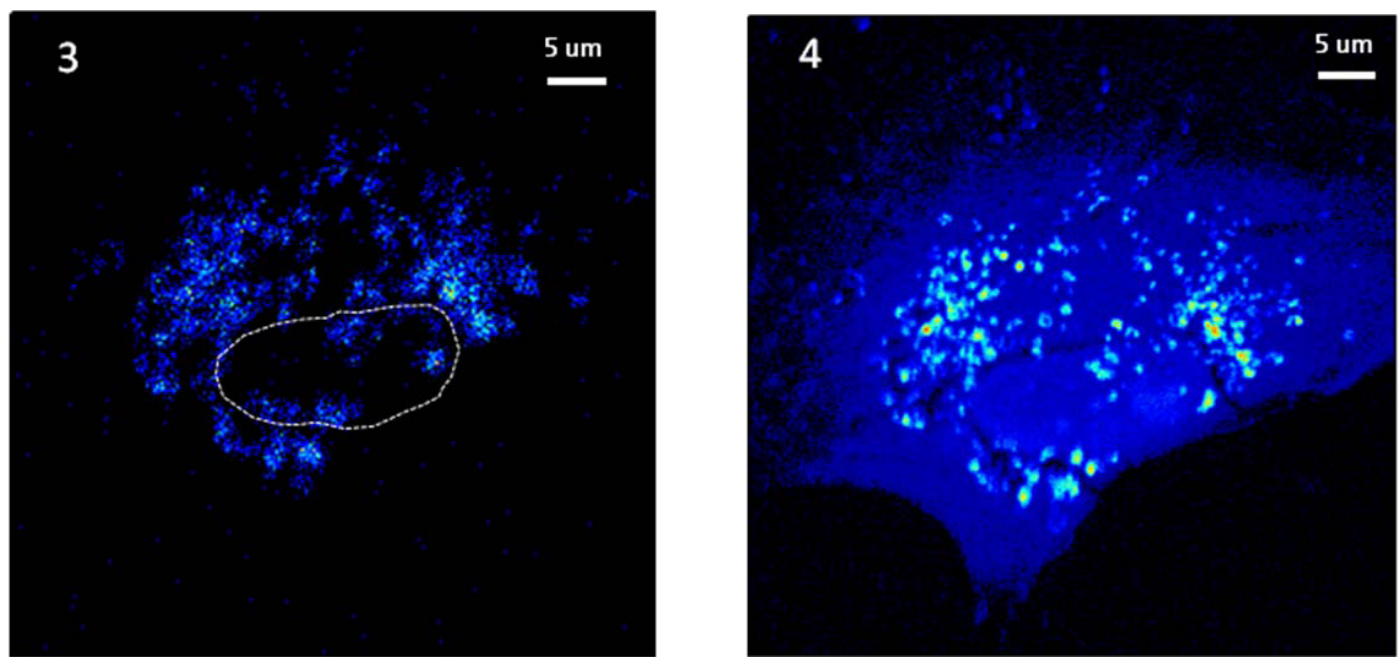

Fig. 3: RBS image of elemental gold; Fig. 4: Off-axis STIM of cell with gold nanoparticles. 